OPEN ACCESS

Edited by: Esteban A. Hernandez-Vargas, Frankfurt Institute for Advanced Studies, Germany

Reviewed by:

Denis Roy,

Laval University, Canada

Axel Kornerup Hansen,

University of Copenhagen, Denmark

${ }^{*}$ Correspondence:

Yibin Feng

yfeng@hku.hk

Specialty section:

This article was submitted to Systems Microbiology, a section of the journal

Frontiers in Microbiology

Received: 04 October 2019

Accepted: 30 January 2020

Published: 21 February 2020

Citation:

Xu Y, Wang N, Tan H-Y, Li S, Zhang $C$ and Feng $Y$ (2020) Function of Akkermansia muciniphila in Obesity: Interactions With Lipid Metabolism, Immune Response and Gut Systems.

Front. Microbiol. 11:219

doi: 10.3389/fmicb.2020.00219

\section{Function of Akkermansia muciniphila in Obesity: Interactions With Lipid Metabolism, Immune Response and Gut Systems}

\author{
Yu Xu, Ning Wang, Hor-Yue Tan, Sha Li, Cheng Zhang and Yibin Feng* \\ School of Chinese Medicine, Li Ka Shing Faculty of Medicine, The University of Hong Kong, Pokfulam, Hong Kong
}

Obesity and its metabolic syndrome, including liver disorders and type 2 diabetes, are a worldwide epidemic and are intimately linked to diet. The gut microbiota interaction has been pointed to as a hot topic of research in the treatment of obesity and related metabolic diseases by influencing energy metabolism and the immune system. In terms of the novel beneficial microbes identified, Akkermansia muciniphila (A. muciniphila) colonizes the mucosa layer of the gut and modulates basal metabolism. A. muciniphila is consistently correlated with obesity. The causal beneficial impact of $A$. muciniphila treatment on obesity is coming to light, having been proved by a variety of animal models and human studies. A. muciniphila has been characterized as a beneficial player in body metabolism and has great prospects for treatments of the metabolic disorders associated with obesity, as well as being considered for next-generation therapeutic agents. This paper aimed to investigate the basic mechanism underlying the relation of A. muciniphila to obesity and its host interactions, as identified in recent discoveries, facilitating the establishment of the causal relationship in $A$. muciniphila-associated therapeutic supplement in humans.

Keywords: Akkermansia muciniphila, obesity, lipid modulation, nutrition therapy, immunity

\section{INTRODUCTION}

Gut microbes have been reported to play a vital role in the regulation of host metabolism in both human and animal studies (Sonnenburg and Backhed, 2016). Host intestine microbiota comprising tens of trillions of microorganisms and more than 1000 different bacteria species have been identified, with at least 3 million genes. The species classification of gut community members is enormous because the distribution and composition of gut microbiota are varied at different intestine anatomical sites and are prone to be influenced by external and internal factors, including lifestyle, diet, and body condition (Clemente et al., 2012). The layout and dysbiosis of gut microbiota have been described to have close pathological and physiological interactions with obesity and metabolic syndrome (Carding et al., 2015). For example, Leptin ${ }^{\mathrm{ob} / \mathrm{ob}}$ obese mice exhibited a significant decrease in the level of Bacteroidetes and a proportional increase in Firmicutes abundance in intestine bacteria, which spurred further investigation of the link between the gut microbiota and obesity (Ley et al., 2005). In subsequent studies, WT C57BL/6J mice were fed either a chow diet or a western diet. It was demonstrated that diet-induced obesity 
could lead to a marked decrease in some Bacteroidetes spp. and increased numbers of Firmicutes, which was consistent with the observation in the genetic obese animal model (Turnbaugh et al., 2008). A high-fat diet (HFD) could cause shifts in the abundance of dominant gut microbiota, including the phylum Proteobacteria as well as Firmicutes, followed by Lactobacillus gasseri and Bifidobacterium spp., both of which were less abundant (Wang and Jia, 2016). In human studies, a lower population of Bacteroidetes in combination with a higher proportion of Firmicutes than in lean control has been detected in obese patients (Nehra et al., 2016). Interestingly, these changes could be reversed by bariatric surgery or dietary intervention. More specifically, the gut microbiota has been proved to have a necessary capacity to harvest energy from the diet and take action on the pathophysiology of obesity via regulating glucose or lipid metabolism (Sonnenburg and Backhed, 2016). Particularly, Akkermansia muciniphila (A. muciniphila) found in the healthy human fecal specimen, has been shown to be one of the few dominant bacteria that have been intensively implicated in the development of obesity (Ottman et al., 2017b).

A. muciniphila has been classified as the sole gram-negative emblematic Verrucomicrobia (Belzer and De Vos, 2012), which is extensively present in the human intestinal mucosa (Huang et al., 2015). Since Derrien et al. (2004) identified the type strain of A. muciniphila as MucT (ATCC BAA-835 1/4 CIP107961T) (Zhai et al., 2018), gene sequencing analysis has shown that there are a lot of mucinase-encoding gene candidates and that its single chromosome contains 2176 genes with a 55.8 percentage of GC content. This oval-shaped and non-motile microorganism is a strict anaerobe and chemo-organotroph that can tolerate low oxygen concentrations (Brodmann et al., 2017). A. muciniphila was capable of producing mucin-degrading enzymes and utilizing mucins as a nitrogen and carbon source in the mucus layer of epithelium. A. muciniphila decomposed these substances into acetic and propionic acid (Huang et al., 2015; Ottman et al., 2016, 2017a) and released sulfate via mucin fermentation. Based on the analysis of its characteristic 16SrRNA signature, A. muciniphila constitutes 3 to 5 percent of the gut microbial community in healthy adults, but this level varies according to many factors. A. muciniphila has shown a close relationship with age in healthy humans. Its colonization starts from early childhood and reaches a similar level to adults, ranging from 5.0 to $8.8 \log$ cells/g, in a year, but it is reduced in the elderly (Collado et al., 2007, 2008; Wang, 2011). Moreover, The presence of $A$. muciniphila and the mucosal pathology differed in patients with metabolic diseases, and they have been inversely associated with the severity of appendicitis and inflammatory bowel disease (Swidsinski et al., 2011). Moreover, the level of gut $A$. muciniphila showed a negative correlation with diabetes, obesity, and other metabolic syndromes (Dingemanse et al., 2015; Remely et al., 2016; Sergey et al., 2017). The dual regulation between $A$. muciniphila and metabolic diseases has shown that not only can the abundance of A. muciniphila be influenced by the presence of pathology but also supplementation of A. muciniphila can have effects on the host body. The propagation of A. muciniphila in the early gut microbiota colonization by early life treatment with vancomycin could have beneficial effects on controlling the development of autoimmune diabetes (Hansen et al., 2012). Oral administration of A. muciniphila at the dose of $2 \times 10^{8}$ bacterial cells per day could reverse HFDinduced obesity in mice by mediating adipocyte metabolism and gut barrier function without influencing food intake (Cani and de Vos, 2017). This preliminary research provided essential evidence that $A$. muciniphila may be considered a promising new therapeutic agent for obesity. At present, the majority of research has demonstrated the beneficial impact of $A$. muciniphila in the prevention and amelioration of metabolic disorders and obesity. And type 2 diabetes mellitus (T2DM) has been characterized as featuring a lower level of A. muciniphila, lowgrade inflammation, and gut permeability disruption (Pascale et al., 2019). The enrichment degree of A. muciniphila can be used as an indicator to evaluate body metabolic status, including glucose homeostasis, serum lipids, and the adipocyte distribution in human (Dao et al., 2016). However, the detailed mechanism of the association between $A$. muciniphila and obesity development has not yet been completely elucidated. Hence, we have reviewed the latest research concerning the role of $A$. muciniphila in obesity and gained an insight into its action on distinctive expression changes of pathways involved in metabolic homeostasis.

\section{METHODS}

We made a comprehensive search of research and reviews in journals about gut microbiota and obesity, but not conference papers or reports. When we entered our school library website, the library provided the most relevant databases containing a wide range of key terms including gut microbiota, obesity, human, and A. muciniphila. Both the United States National Library of Medicine National Institutes of Health (PubMed) and the Web of Science databases include publications from major academic publishers like Elsevier, Springer, Taylor and Francis, etc. We searched both on PubMed and the Web of Science, retrieved manuscripts and removed duplicate manuscripts. Based on the title and abstract, we excluded some unrelated citations and retrieved 146 full-text articles for further evaluation.

\section{RESULTS}

\section{Influence of Akkermansia muciniphila on Lipid Desregulation in Obesity}

Energy consumption promotion has been considered an effective method for body weight loss. Pharmacotherapy, diet, and lifestyle intervention improve energy metabolism, of which the related mechanisms have been proved to involve interaction with gut microbiota (Zhang et al., 2019). As gut microbiota dysbiosis is a contributing factor to energy imbalance, gut microbiota intervention is a potential therapeutic method for treating obesity-related metabolic diseases, including hyperlipidemia and hyperglycemia (Kim et al., 2019). The relationship between A. muciniphila and the host is reflected in the energy consumption associated with glucose and lipid metabolism (Zhang et al., 2019) and thereby affecting the development of 
obesity. Previous studies proved a close negative link between the enrichment of $A$. muciniphila and the development of obesity, in that its abundance is inversely proportional to the bodyweight of animals and humans. Replenishment with A. muciniphila in obese mice reversed high fat diet-induced metabolic disorders such as metabolic endotoxemia, fat-mass gain, and insulin resistance (Everard et al., 2013). However, oral administration of $A$. muciniphila did not change plasma lipid in normal chow diet-fed mice (Li et al., 2016). This observation indicated that $A$. muciniphila only showed its modulation effect in the context of lipid metabolic disorder. In cAMP-responsive binding protein $\mathrm{H}(\mathrm{CREBH})$ deficiency-induced hyperlipidemia mice, A. muciniphila inoculation could cause clearance of triglyceride and postprandial chylomicrons to avoid acute lipid overload in the circulation. During the clearance progress, the expression of low-density lipoprotein (LDL) receptors was increased, which facilitated the upregulation of intermediatedensity lipoprotein (IDL) via the induction of apolipoprotein $B$ 100 and apolipoprotein E (Shen et al., 2016; Bannerman et al., 2018). Thus, replenishment with A. muciniphila can ameliorate western diet-induced atherosclerotic lesion aggravation in Apolipoprotein E deficient (Apoe ${ }^{-/-}$) mice. Although the colonization of live A. muciniphila did not result in the alteration of serum total cholesterol (TC), total triglyceride (TG), high density lipoprotein (HLD), and LDL in Apoe - $^{-}$ mice, the soluble receptor for tumor necrosis factor type II (sTNFRII) was significantly reduced, which led to improvement in atherosclerosis and congestive heart failure (Li et al., 2016). The metabolic activity of A. muciniphila on the host metabolic physiology has also been determined to interact directly with several lipid metabolic substances, including changing the endotoxin level (Ozkul et al., 2017) and short-chain fatty acid (SCFA) production (Ottman et al., 2017b), as well as increasing fatty acid oxidation in the intestine and adipose tissue (Lukovac et al., 2014). As a producer of SCFAs, A. muciniphila has been reported to convert dietary fiber into acetate, propionate, and butyrate; these metabolites can have effects on glucose and lipid homeostasis (Chambers et al., 2018). The SCFA production in the distal ileum induced the action of the various transcription factors to control lipid metabolism and growth; for example, propionate and butyrate stimulation led to an obvious increase in fasting-induced adipocyte factor (Fiaf) production and decreased Gpr43, histone deacetylase (HDAC), and PPAR $\gamma$ expression. All these three SCFAs (acetate, propionate, and butyrate) significantly increased $\mathrm{Hdac} 3$ and $\mathrm{Hdac} 5$ production in intestinal epithelial organoids (Lukovac et al., 2014). The recognition of the beneficial impact of $A$. muciniphila on metabolic disorders during obesity provoked the further investigation of internal cytokines or external metabolites to make a clear statement on the metabolic activities of $A$. muciniphila, particularly with regards to some novel bioactive substances (Schneeberger et al., 2015). For example, compared with T2DM patients, an increased level of A. muciniphila-derived extracellular vesicles (AmEVs) was found in the fecal samples from healthy human. Meanwhile, AmEVs reduced body weight, improved glucose tolerance, and ameliorated gut permeability in HFD-induced diabetic mice, as well as enhancing tight junctions via upregulating the expression of occludin in lipopolysaccharide (LPS)-induced Caco-2 cells (Chelakkot et al., 2018). Since A. muciniphila has been considered to be a promising prebiotic in the improvement of metabolic syndrome, viable $A$. muciniphila have been implemented for metabolic disorder treatment (Ouwerkerk et al., 2017). Pasteurization is an effective way to make A. muciniphila safe for use. Researchers have found that pasteurization enhanced the capacity of $A$. muciniphila to reduce fat mass and improve dyslipidemia (Plovier et al., 2017; Anhe et al., 2019). This enhancement had a close connection with the host intestinal energy metabolism and promoted its capacity to reverse the high expression of Fmo3, which modulated TMA conversion into TMAO. In more detail, this action was associated with the interaction of Toll-Like Receptor 2 signaling via Amuc_1100, a specific protein located at the outer membrane of $A$. muciniphila (Plovier et al., 2017). Amuc_1100 has been proved to modulate the gut barrier and intestinal permeability via tight-junction proteins such as Occludin (Ocln), Claudin 3 (Cldn3), and Cannabinoid Receptor 1 (Cnr1) (Li et al., 2016).

\section{The Causative Role of Akkermansia muciniphila in Liver Disorder in Obesity}

Liver disorder disease is a complicated metabolic disease and commonly shows a close relationship with obesity as well as the related gut microbiota changes in obesity (Duranti et al., 2017). In gut microbiota transplantation studies, germ-free C57BL/6J mice receiving intestinal bacteria from HFD-fed mice with high blood glucose were prone to develop liver steatosis and insulin resistance compared with counterparts that were transplanted with bacteria from healthy mice (Aron-Wisnewsky et al., 2013). Different degrees of liver pathology have also been linked with the different varieties of gut microbiota (Meng et al., 2018). Human studies have indicated that the abundance of Bacteroidetes in nonalcoholic fatty liver disease patients is much lower than that in simple steatosis and healthy groups (Fukui, 2015). Compared with healthy adults, an apparent decline has been shown in the abundance of $A$. muciniphila in patients with alcoholic steatohepatitis (Van Best et al., 2015). Collective findings have confirmed that the action of $A$. muciniphila could extend to various hepatic disorders such as fatty liver disease, hepatic inflammation, and hypercholesterolemia. Oral supplementation of A. muciniphila can restore A. muciniphila depletion caused by ethanol exposure in mice, preventing the liver from alcoholic injury, neutrophil infiltration, and steatosis (Grander et al., 2018). In a concanavalin A-induced liver injury mouse model, oral administration of $A$. muciniphila reduced serum Alanine Aminotransferase (ALT) and Aspartate Aminotransferase (AST) and alleviated liver damage (Wu et al., 2017). Mechanistic study revealed that $A$. muciniphila improved epithelial barrier function via fluorescein isothiocyanate-dextran translocation (FD4) in mucosa to elevate gut status. A. muciniphila treatment restored epithelial tight junction proteins (claudin-3 and occludin) in colonic epithelial cells to defend against the invasion of ethanol. The evidence suggests that $A$. muciniphila has protective actions against alcoholic liver disease focusing on the intestine (Grander et al., 2018). Moreover, the interference of A. muciniphila in 
improving intestinal barrier function is evoked by improving occludin and Tjp-1 expressions and suppressing LPS production followed by increasing the variety and volume of gut microbes (Wu et al., 2017). Above all, as shown in Figure 1, during the development of lipid and liver disorders, A. muciniphila modulated the lipid metabolism in circulation, including adipose, liver, and intestine, and the internal metabolite changes caused by A. muciniphila were also involved in these actions.

\section{Regulation of the Inflammatory Response in Obesity by Akkermansia muciniphila}

Obesity and associated metabolic disorders are characterized by a low-grade inflammatory response. Previous reports have documented that antibiotic or probiotic carbohydrate treatment modulated gut microbiota, leading to a reduction in metabolic endotoxemia that induced low-grade inflammation. The anti-inflammatory process is associated with lower intestinal permeability in diet-induced obesity mice via improving the tight-junction integrity (Cani et al., 2008), and it has been confirmed that this process might control and increase the production of endogenous GLP-2 in Leptin ${ }^{\mathrm{ob} / \mathrm{ob}}$ mice (Cani et al., 2009). The intervention of $A$. muciniphila in germ-free mice suggested the existence of host- $A$. muciniphila interaction in obesity involving the activation of immunological signaling (De Vos, 2017). This interaction is supported by evidence indicating that $A$. muciniphila was highly enriched in Rag1 ${ }^{\text {tm1Mom }}$ mice deficient in $\mathrm{B}$ and $\mathrm{T}$ lymphocytes compared with wild-type mice (Zhang et al., 2015). A. muciniphila has been proved to have a robust correlation with inflammatory markers and adipose tissue homeostasis, and also with insulin and glycemia at the onset of obesity (Schneeberger et al., 2015). The indirect alteration evoked by A. muciniphila in obesity also involved the stimulation of Treg cell proliferation (Shin et al., 2014). The triglyceride clearance action of $A$. muciniphila in CREBH-null mice was mediated by LDL receptor signaling, which further involved the suppression of hepatic ER stress marker-glucose regulated protein 94 and inflammatory transcription factors Junamino-terminal kinase 1 (JNK1) and JNK2 (Shen et al., 2016). Accumulating evidence concerning the correlation between gut permeability and LPS absorption has provided reliable support the identified association with metabolic inflammation (Cani et al., 2012). A. muciniphila restored the gut barrier function via decreasing LPS evoked by a western diet (Kelly et al., 2015). The amelioration of LPS-induced artic and systemic inflammation suppressed the macrophage transmigration into intima and adhesion onto endothelium, as evidenced by the reduced expressions of ICAM-1, MCP-1, F4/80, TNF- $\alpha$, and IL-1 $\beta$ (Anonye, 2017; Wang et al., 2017). Colonization of A. muciniphila in an immune-mediated liver injury model restrained the increased LPS level and substantially decreased the expression of serum pro-inflammatory markers (interleukin(IL)2, Interferon- $\gamma$ (IFN- $\gamma$ ), IL-12p40, MCP-1, MIP-1a, and MIP-b), as well as attenuating the hepatocellular apoptosis by the Tolllike receptor-4 (TLR-4) pathway (Wu et al., 2017). MyD88/TLR2 innate immune signaling also appeared to be mediated by
A. muciniphila, and A. muciniphila consistently evoked a lower level of serum endotoxin and islet toll-like receptor, as well as promoting the secretion of antimicrobial peptide $\operatorname{Reg} 3 \gamma$ in colon, which caused bacterial remodeling in non-obese diabetic mice (Hanninen et al., 2018). Interferon- $\gamma$ (IFN $\gamma$ ), as a key cytokine of the immune system in glucose metabolism, could be negatively modulated by $A$. muciniphila, which led to the improvement of glucose tolerance. The IFN $\gamma$-modulated gene Irgm 1 has been identified in the mouse ileum and can regulate the abundance of A. muciniphila (Greer et al., 2016). However, $S$. Typhimurium-induced inflammation could turn the commensal bacterium into a pathobiont. A. muciniphila can exacerbate $S$. Typhimurium-induced inflammation, coinciding with higher mRNA levels of IFN $\gamma$ and IFN $\gamma$-induced protein 10 (IP-10), tumor necrosis factor- $\alpha$ (TNF- $\alpha$ ), and interleukin (IL)- 6 and IL17 , but not IL-18. This inflammatory exacerbation was found in a gnotobiotic mouse model colonized with a defined simplified microbiota of eight bacterial species (Zhai et al., 2015; Pleasure et al., 2018). The presence of A. muciniphila enhanced the infection with $S$. Typhimurium and promoted macrophage infiltration into cecal tissue. A. muciniphila at extremely high cell concentrations of $10^{6}$ bacteria/ml can stimulate interleukin 8 (IL-8) secretion on enterocytes. This means that the low level of pro-inflammatory stimulation evoked by $A$. muciniphila seemed unable to insult a powerful inflammatory cascade. Conversely, the low-level stimulation involved LPS production may keep the mucosa-associated immune system alerted at an appropriate level (Reunanen et al., 2015). It is likely that, normally, A. muciniphila shows a protective role in intestinal immunity but that the role of $A$. muciniphila in the ecosystem can be changed in response to the presence of inflammation (Ganesh et al., 2013). Despite the fact that there is no clear definition of the appropriate levels that can describe a shift between a pathological and a healthy condition, below a given amount of A. muciniphila, human subjects were less likely to have a positive response in terms of improved inflammatory cytokines and insulin resistance (Schneeberger et al., 2015). In contrast to the adverse effect of A. muciniphila in the development of DSSinduced colitis, A. muciniphila-derived extracellular vesicles, as a mediator on intestine immunity, have shown an obvious protective function against the progression of DSS-induced colitis (Kang et al., 2013). To some degree, the host regulation of A. muciniphila contributed to its derived materials, including pililike proteins (Ottman et al., 2017c). The outer membrane pililike protein of A. muciniphila (Amuc_1100) directly modulated the gut immunity and increased trans-epithelial resistance, which enhanced the capacity of A. muciniphila to improve host metabolic homeostasis (Ottman et al., 2017c).

\section{Interaction of Akkermansia muciniphila With the Gut Ecosystem and Homeostasis in Obesity}

Certainly, to determine whether the gut microbiota contributes to maintaining a healthy status, the variation in the host micro-environment ecosystem should not be overlooked (Lin et al., 2014). Poor diversity of the gut ecosystem can result 


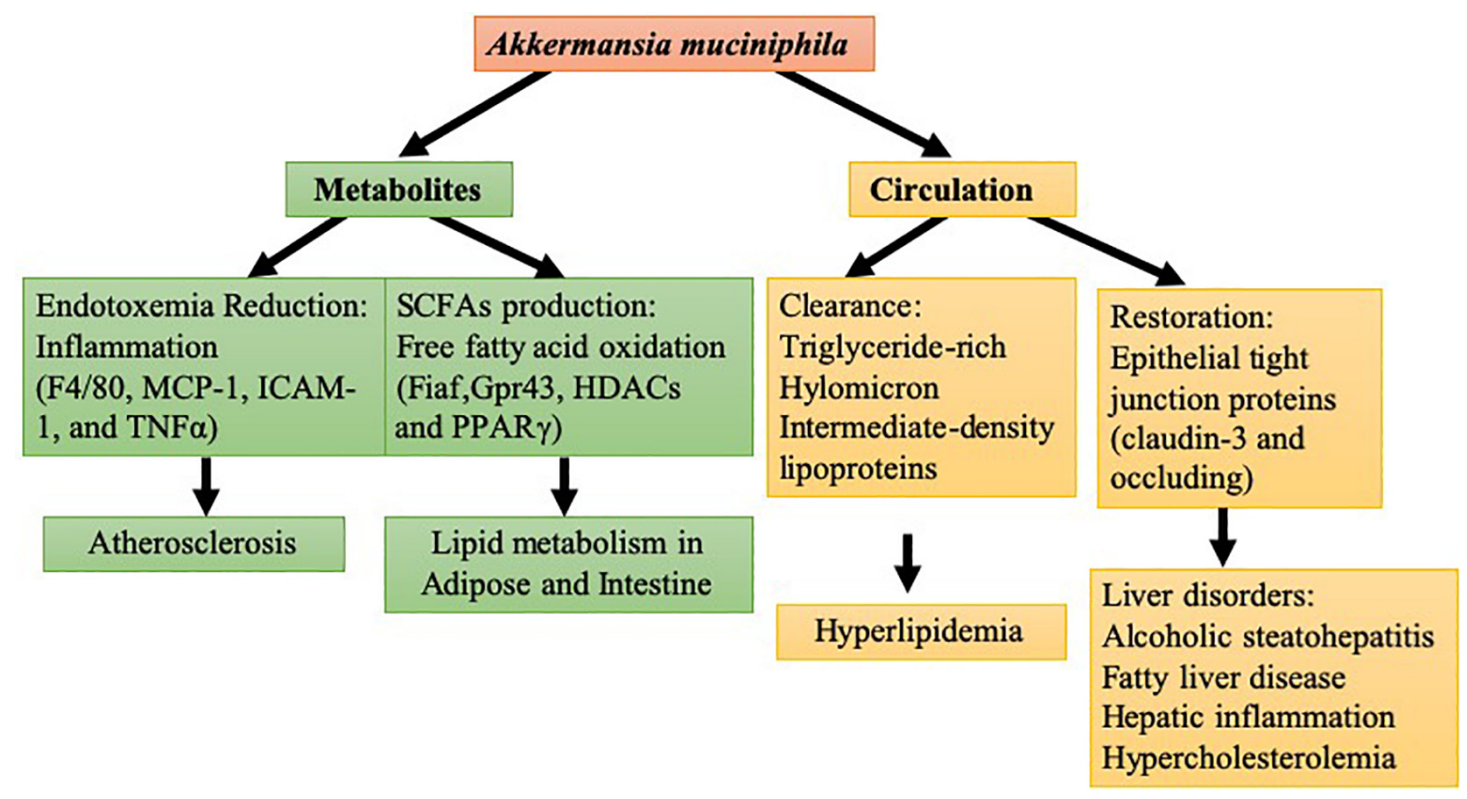

FIGURE 1 | Molecular modulation of $A$. muciniphila in lipid and liver disorder: in yellow are the circulation mechanisms of $A$. muciniphila in hyperlipidemia and liver disorder. In green are the metabolite mechanisms of $A$. muciniphila in lipid metabolism in tissue and atherosclerosis.

in the alteration of intestinal permeability and the breakdown of the intercellular junctions between mucosal cells, both of which permit the diffusion and activation of potentially immune substances from intestinal content into an inflammatory state, causing a disorder of the gut tract. It has been reported that mucins constitute a key ecological niche that shapes the microbiota composition. Supplementation of mucins had a positive impact on the abundance of Akkermansia, Bacteroides, and Ruminococcus (Van Herreweghen et al., 2018), while higher simulated colonic $\mathrm{pH}$ showed less positive effects on the level of Akkermansia. In addition to the nutritional resources and environmental factor modulation on the gut microbiome, the extracellular mucin degradation by Akkermansia could provide growth benefits to gut community species via trophic interactions (Belzer et al., 2017; Zhang S. et al., 2018). The mucolytic activity of A. muciniphila could produce mucin-degrading enzymes (proteases, glycosyl hydrolases, and sulfatases), oligosaccharides and short-chain fatty acids (SCFAs, including lactate and acetate), all of which juxtaposed community members could utilize for their own proliferation. For example, the mucolytic enzymes induced by A. muciniphila increased upon the presence of a butyrogenic gut commensal, Anaerostipes caccae (Chia et al., 2018). Furthermore, the monosaccharides supported by A. muciniphila promoted the proliferation of Faecalibacterium prausnitzii, which converted acetate and lactate into butyrate (Lopez-Siles et al., 2018). It has been shown that A. muciniphila and $F$. prausnitzii, co-occurring in the mucosa, had a syntrophic relationship with each other and that both species were reduced in inflammatory bowel disease (Lopez-Siles et al., 2018). The metabolites were induced by $A$. muciniphila, even though not completely, and thus created metabolic cross-feeding interactions in the gut ecosystem. Moreover, the enrichment of A. muciniphila promotes microbial gene richness and microbial ecosystem abundance, including Firmicutes, Bacteroidetes, Actinobacteria, and Euryarchaeota (Dao et al., 2016). A. muciniphila can form an alliance with Bacteroides-Prevotella, Bacteroidetes, Firmicutes, and Lactobacillus, so after the colonization of A. muciniphila, the elevated abundance of $A$. muciniphila and these allies indicates that the protective effects have also altered gut microbe community composition. The colonization of A. muciniphila in mice also evoked a global transcriptional host response, which revealed a unique, consistent, and site-specific regulation of 750 genes (Derrien et al., 2011). The intestinal epithelium is the mediator of gut microbe and gut barrier function, and the gut barrier status is directly reflected by mucus layer alteration and the changes in immune mediators secreted by host tissue. The interaction with colonic mucin caused by $A$. muciniphila has been elevated at the mucin microarray platform, and it had shown strong mucin binding capacity compared with Desulfovibrio spp. (Earley et al., 2015). Specifically, oral A. muciniphila administration as a prebiotic treatment for diet-induced obesity mice not only increased Muc2 protein production to restore mucus layer thickness but also prevented the intestinal barrier from injury via decreasing high fat-induced endotoxemia (Everard et al., 2013). A. muciniphila is located in close relation to the intestinal cells. Its adherence to the enterocyte cells in the intestinal tract strengthens the integrity of this epithelial cell layer, resulting in the activation of the intestinal immune system and resistance to leaky gut (Derrien et al., 2011; Reunanen et al., 2015). Moreover, A. muciniphila reduced gut permeability by improving the inner mucin layer thickness of ileum in $\mathrm{Apoe}^{-/-}$ mice, which involved increased expressions of Occludin and ZO-1 (Li et al., 2016). In addition, the strong adhesion of A. muciniphila to Caco-2 and HT-29 human colonic cell lines 
helped to strengthen the integrity of enterocyte monolayer via increasing the transepithelial electrical resistance (Reunanen et al., 2015). A. muciniphila can secrete some enzymes into the gut tract to regulate the mucin protein on the mucosa layer (Geerlings et al., 2018). The related microbiota on the mucosa layer and its associated composition have beneficial effects on protecting the intestine epithelial cells from injury. Cross-talk in gut- $A$. muciniphila interactions and the associated improvement in obesity disease are shown in Figure 2. It has been shown through pathway reconstructions that alteration of mucosal gene profiles by colonization by A. muciniphila is involved in an increase in expression immune response- and cell fate determination-related genes (Derrien et al., 2011). The colonization of $A$. muciniphila in germ-free mice altered the mucosal gene expression, according to the immune pathway reconstruction, which indicated that the pathways modulated by $A$. muciniphila involved mucus homeostasis for immune tolerance toward commensal microbiota and basal metabolism (Derrien et al., 2011).

\section{NUTRITIONAL AND MEDICINAL APPROCHES FOR MODULATION OF Akkermansia muciniphila}

As the major environmental factor for intestinal microbiota, dietary intervention, as a healthy lifestyle approach to treating obesity, has been proved to be beneficial for intestine homeostasis and to be strongly associated with a remarkable change in A. muciniphila abundance in the gut (Anhe et al., 2016). Dietary interventions for reducing obesity have been suggested that focus on calorie restriction, energy reduction, or a diet rich in prebiotic fibers, all of which are associated with an increased level of A. muciniphila in the host (Verhoog et al., 2019). For example, clinical trials have shown a significant increase in A. muciniphila in a calorie restriction study of 49 overweight and obese adults (Stenman et al., 2016). Diets rich in prebiotic fiber increased the number of $A$. muciniphila in the microbiome (Gomez-Gallego et al., 2016). Meanwhile, direct probiotic supplements can cause a $>100$-fold enrichment in the level of A. muciniphila (Everard et al., 2013; Zhao et al., 2017). Pomegranate extract promoted the growth of $A$. muciniphila in vivo and led to the formation of ellagic acid (Wang et al., 2016; Henning et al., 2017). Furthermore, a review indicated that the metabolic benefits of polyphenol-rich extracts, such as cranberry extract, curcumin, and epigallocatechin gallate, contribute to the prevention of weight gain and obesity-related metabolic disturbances through increasing the abundance of this bacterial species in the gut microbiota. In line with these findings, the molecular mechanism by which cranberry extract increased mucin 2 secretion has been explored; it might supply ample trophic resources for $A$. muciniphila to thrive and might lead to improved gut mucous layer in mice (Pierre et al., 2013). Moreover, a butter fat-based diet supplemented with California table grape powder can reduce obesity and hepatic lipogenesis in mice, associated with an increase in A. muciniphila in the gut (Baldwin et al., 2016). A higher abundance of A. muciniphila caused by the anti-obesity effects of Capsaicin also induces the increased expression of anti-microbial protein Reg3g and Mucin 2 protein in the gut (Shen et al., 2017). Compared with healthy patients, metformin promoted the abundance of $A$. muciniphila in diabetic patients. A. muciniphila further contributed to the anti-diabetic effects of metformin via enrichment of mucin degradation and SCFA secretion in type 2 diabetes patients (De La Cuesta-Zuluaga et al., 2017). Dietary polyphenols have shown protection against HFD-induced obesity through modification of the gut microbiota community. Oral administration of a steroidal saponin extract from Agave salmiana in HFD mice showed anti-obesity effects, attenuating hepatic steatosis, and increased the population of $A$. muciniphila in a dose-dependent manner (Leal-Diaz et al., 2016). Dietary polyphenols resulted in a reduced inflammatory response in the intestine and led to an improvement of metabolic syndrome. Other dietary polyphenols such as cranberry polyphenol extract have shown anti-obesity effects associated with promoting the growth of A. muciniphila via downregulation of inflammatory cytokines such as TNF- $\alpha$ and IL-6 (Roopchand et al., 2015). However, the mechanism underlying the effect of these polyphenols in promoting the abundance of A. muciniphila is still unclear, and further studies are needed to confirm whether these effects on gut microbiota are mediated or direct (Spanogiannopoulos et al., 2016). However, it has recently been proved that black tea- or grape-derived polyphenols can directly upregulate the expansion of A. muciniphila (Etxeberria et al., 2015), further work is needed to find the underlying mechanism involved (Ozdal et al., 2016). In conclusion, the content of A. muciniphila in the microbiome can be enhanced by medical and nutritional therapy, including diet, medicine, prebiotics, and probiotics (Duparc et al., 2017), The ease of A. muciniphila supplementation through nutritional therapy indicates that these actions might be an effective method for the treatment of metabolic diseases. Still, not all medicinal nutrition can cause increased A. muciniphila, for example, grape proanthocyanidins do not (Zhang L. et al., 2018).

\section{DISCUSSION AND CONCLUSION}

Consistent findings from both preclinical and clinical research have revealed declined abundance of $A$. muciniphila in obesity and metabolic syndromes. With more preclinical and clinical research focused on $A$. muciniphila, the molecular mechanism of $A$. muciniphila for treating metabolism is becoming more apparent. It is worth looking forward to finding the systematic regulatory mechanism of A. muciniphila in rodents to provide essential evidence for human research; hopefully, these achievements can be translated to human intervention research, though human studies still hold uncertain ethical and inevitable practical limitations. From the schematic overview of the metabolic activities of $A$. muciniphila in intestine and interaction with the host as a result of $A$. muciniphila colonization (Figure 3), conclusions can be drawn. Oral gavage with $A$. muciniphila or the beneficial dietary polyphenol increased the abundance of intestinal $A$. muciniphila to a level sufficient 


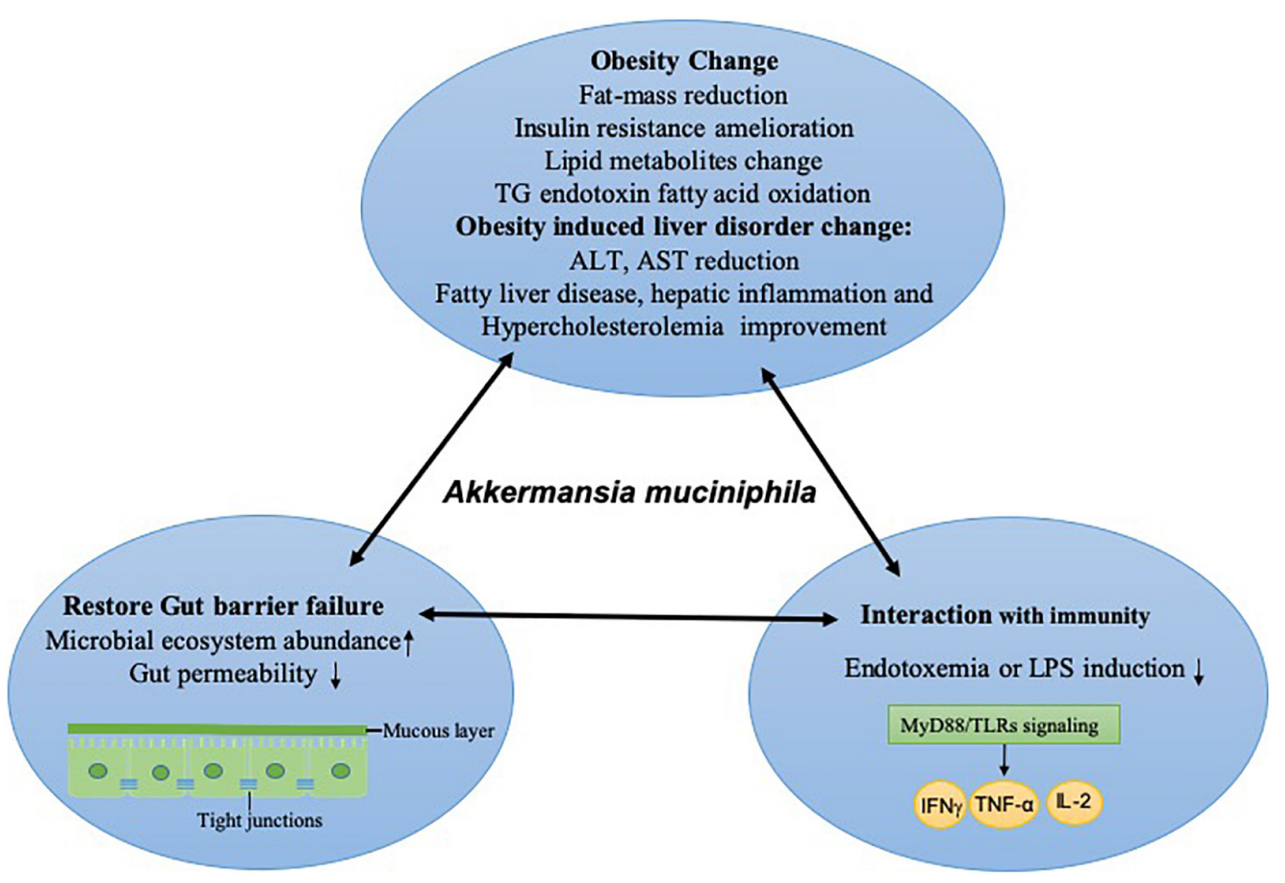

FIGURE 2 | Cross talk in host-A. muciniphila interactions and the associated improvement in obesity disease. After the colonization of $A$. muciniphila, the state of homeostasis and symbiotic relationships is improved by immunity activity. This state includes obesity and its associated liver disorder changes in the host; the gut barrier is restored either physically (at the mucous layer or the level of tight junctions) or at the level of the gut ecosystem, and reduced inflammatory responses are often induced by endotoxemia or LPS via TLRs and downstream cytokines, which improve intestinal permeability. TG, total triglyceride; ALT, Alanine Aminotransferase; AST, Aspartate Aminotransferase; LPS, lipopolysaccharide; TLR, Toll-like receptors; IFN $\gamma$, Interferon- $\gamma$; TNF, tumor necrosis factor, IL-2, interleukin-2.

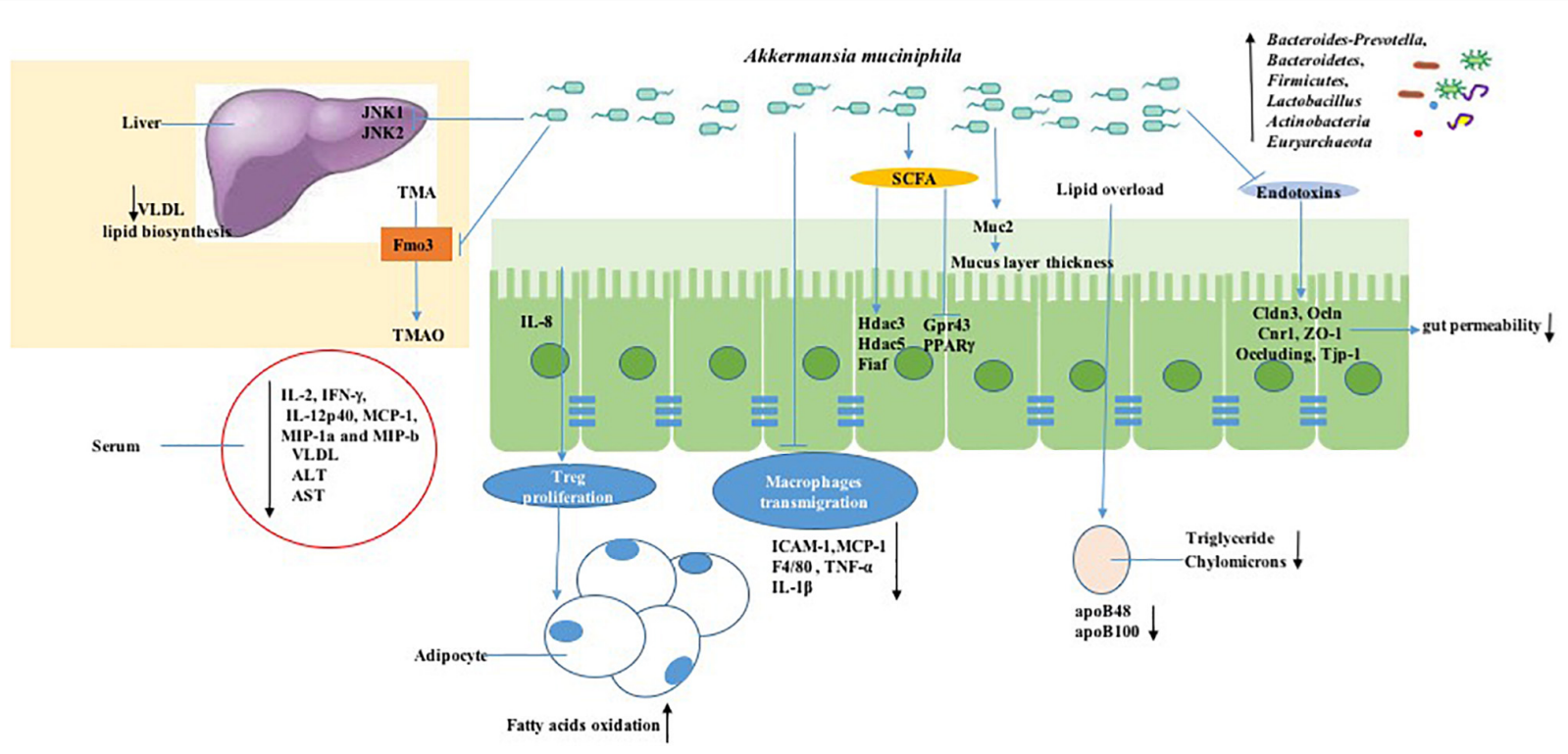

FIGURE 3 | Mechanisms of signaling due to A. muciniphila action in obesity. A. muciniphila can decrease the serum level of inflammatory cytokines such as IL-2, IFN- $\gamma$, IL-12p40, and MCP-1. Meanwhile, A. muciniphila decreases the lipid overload process associated with the LDL receptor pathway by decreasing apoB48 and apoB100 on LDLs. Moreover, short-chain fatty acid production stimulated by $A$. muciniphila is involved in signaling to the host by inhibiting histone deacetylase (HDAC) or by activating G-protein-coupled receptors, which triggers other metabolic pathways, together resulting in immune stimulation including macrophage transmigration and Treg proliferation changes. A. muciniphila regulates the intestinal permeability and gut barrier by tight-junction proteins such as Claudin 3 (Cldn3), Occludi(Ocln), and Cannabinoid Receptor 1 (Cnr1) and reverses the high enzyme expression of flavin-containing monooxygenase 3 (FMO3), which modulates TMA conversion into TMAO in the liver. 
to reduce systemic inflammation, improve gut disruption, and contribute to the steady state of the host basal metabolism and the promotion of other beneficial microbes via various signaling pathways. This suggests that therapeutic intervention focusing on A. muciniphila in intestinal microbiota could be considered as a promising strategy for the prevention and treatment of obesity and metabolic disorder diseases, including cardiovascular disease, liver injury, and type II diabetes mellitus (Zhang L. et al., 2018). These statements can be supported by the evidence, as shown in Table 1, which shows an overview of Akkermansia and its associated biomarkers in clinical studies of obesity. A recent clinical trial (NCT02637115) indicated that 3-month oral administration of A. muciniphila in obese patients is safe and well-tolerated. Meanwhile, supplementation of A. muciniphila decreased the body weight and improved liver dysfunction as well as inflammation in patients, as shown in a double-blind, randomized human study (Depommier et al., 2019). It has been demonstrated that A. muciniphila could be isolated from purified hog gastric mucus (Van Der Ark et al., 2018) and cultured in vivo for medicinal application. However, the development of translational approaches is limited by a lack of specific growth-dependent compounds and suitable culture conditions without oxygen influence (Ouwerkerk et al., 2016). Another reason that the products, including A. muciniphila, are unable to be commercially viable is that their safety assessment cannot reach the requirements of Novel Foods Regulation (Gomez-Gallego et al., 2016). Moreover, though the correlation of gut microbiota alterations and chronic metabolic diseases has been discussed, the consequences or causes of these bacterial changes are still unclear. It is inadvisable to make an absolute statement on the positive effects of A. muciniphila on metabolic diseases. For example,

TABLE 1 | Overview of $A$. muciniphila and its associated biomarkers in clinical studies of obesity.

\begin{tabular}{|c|c|c|c|c|}
\hline Ref. & Participation & $\begin{array}{l}\text { Intervention or } \\
\text { Observation }\end{array}$ & Study Period & Results \\
\hline $\begin{array}{l}\text { (Medina-Vera et al., } \\
\text { 2019) }\end{array}$ & 81 Patients with T2DM & A reduced-energy diet & 3 months & $\begin{array}{l}\text { Dietary portfolio consumption increased levels of } A \text {. muciniphila and } \\
\text { improved glycaemic control, dyslipidaemia, and inflammation. }\end{array}$ \\
\hline $\begin{array}{l}\text { (Roshanravan et al., } \\
\text { 2017) }\end{array}$ & $\begin{array}{l}60 \text { patients, overweight } \\
\text { and with obese } \\
\text { diabetes }\end{array}$ & $\begin{array}{l}600 \mathrm{mg} / \mathrm{d} \text { butyrate, } \\
10 \mathrm{~g} / \mathrm{d} \text { inulin powder, } \\
\text { both inulin and butyrate } \\
\text { or placebo }\end{array}$ & 45 days & $\begin{array}{l}\text { Supplementation of insulin and butyrate can increase } \\
\text { A. muciniphila, and butyrate decreases TNF- } \alpha \text { mRNA expression, } \\
\text { hs-CRP, MDA, and diastolic blood pressure levels. }\end{array}$ \\
\hline (Walker et al., 2019) & $\begin{array}{l}28 \text { obese men with } \\
\text { metabolic syndrome }\end{array}$ & $\begin{array}{l}1 \mathrm{~g} \text { polyphenol } \\
\text { resveratrol orally twice } \\
\text { daily or placebo }\end{array}$ & 35 days & $\begin{array}{l}\text { Polyphenol resveratrol improves glucose homeostasis and } \\
\text { increases the abundance of } A \text {. muciniphila. }\end{array}$ \\
\hline (Allin et al., 2018) & $\begin{array}{l}134 \text { Danish adults with } \\
\text { prediabetes and } 134 \\
\text { healthy controls }\end{array}$ & Observation & & $\begin{array}{l}\text { The abundance of the mucin-degrading bacterium A. muciniphila } \\
\text { obviously decreased in prediabetes. }\end{array}$ \\
\hline (Dao et al., 2016) & $\begin{array}{l}49 \text { adults, overweight } \\
\text { and obese }\end{array}$ & $\begin{array}{l}6 \text {-week calorie } \\
\text { restriction }\end{array}$ & 12 weeks & $\begin{array}{l}\text { A. muciniphila abundance improved fasting plasma glucose, } \\
\text { plasma triglycerides, and body fat distribution. }\end{array}$ \\
\hline (Khan et al., 2018) & $\begin{array}{l}43 \\
\text { hypercholesterolemic } \\
\text { patients and } 19 \text { healthy } \\
\text { controls }\end{array}$ & $\begin{array}{l}27 \text { patients with } \\
\text { Atorvastatin treatment }\end{array}$ & 2 years & Atorvastatin treatment increased the abundance of $A$. muciniphila. \\
\hline (Liu et al., 2017) & $\begin{array}{l}70 \text { female patients with } \\
\text { T2DM and } 70 \text { healthy } \\
\text { females. }\end{array}$ & Observation & & $\begin{array}{l}\text { Decreased } A \text {. muciniphila was associated with fasting blood } \\
\text { glucose and urine glucose. }\end{array}$ \\
\hline $\begin{array}{l}\text { (Collado et al., 2010, } \\
\text { 2012; Santacruz et al., } \\
\text { 2010) }\end{array}$ & $\begin{array}{l}16 \text { infants with obese } \\
\text { mother and } 256 \text { infants } \\
\text { of normal mothers as } \\
\text { control }\end{array}$ & Observation & & $\begin{array}{l}\text { Prevalence of } A \text {. muciniphila was lower in control infants with } \\
\text { normal mothers. }\end{array}$ \\
\hline $\begin{array}{l}\text { (De La Cuesta-Zuluaga } \\
\text { et al., 2017) }\end{array}$ & $\begin{array}{l}28 \text { participants with } \\
\text { diabetes and } 84 \\
\text { healthy controls }\end{array}$ & Metformin & & $\begin{array}{l}\text { Diabetes patients taking metformin had a higher relative abundance } \\
\text { of } A \text {. muciniphila compared with healthy control. }\end{array}$ \\
\hline (Palleja et al., 2016) & $\begin{array}{l}13 \text { morbidly obese } \\
\text { patients }\end{array}$ & $\begin{array}{l}\text { Roux-en-Y gastric } \\
\text { bypass (RYGB) }\end{array}$ & 12 months & $\begin{array}{l}\text { RYGB changed the relative abundances of } 31 \text { species, including } \\
\text { A. muciniphila, within the first } 3 \text { months. These abundance changes } \\
\text { can be maintained for } 9 \text { months. }\end{array}$ \\
\hline (Brahe et al., 2015) & 53 women with obesity & Observation & & $\begin{array}{l}140 \text { metagenomic species, including A. muciniphila, were } \\
\text { correlated with metabolic markers. }\end{array}$ \\
\hline $\begin{array}{l}\text { (Yassour et al., 2016; } \\
\text { Cortez et al., 2018) }\end{array}$ & 21 patients with T2DM & $\begin{array}{l}\text { Duodenal-jejunal } \\
\text { bypass surgery medical } \\
\text { care }\end{array}$ & 12 months & The level of gut $A$. muciniphila in the surgery group increased. \\
\hline $\begin{array}{l}\text { (Depommier et al., } \\
\text { 2019) }\end{array}$ & $\begin{array}{l}32 \text { participants, } \\
\text { overweight/obese } \\
\text { insulin-resistant }\end{array}$ & $\begin{array}{l}\text { Oral supplementation } \\
\text { of } 10^{10} \mathrm{~A} \text {. muciniphila } \\
\text { bacteria, either live or } \\
\text { pasteurized }\end{array}$ & 3 months & $\begin{array}{l}\text { A. muciniphila decreased body weight and reduced the levels of the } \\
\text { relevant blood markers for liver dysfunction and inflammation, while } \\
\text { the overall gut microbiome structure was unaffected. }\end{array}$ \\
\hline
\end{tabular}


an excessive level of $A$. muciniphila has been found to exist in Parkinson's disease and eczematous infants, and the adverse action of $A$. muciniphila in eczematous infants may cause reduced integrity of the intestinal barrier function, therefore leading to increased risk of eczema development (Zheng et al., 2016). The adverse effect of excessive A. muciniphila in Parkinson's disease is still unclear, but it has been reported that the reduction of SCFAs and the intestinal amyloid deposition evoked by the alteration of gut microbiota are associated with exacerbating cognitive deficits in Parkinson's disease (Zhang et al., 2017). The gut microbiota has also been shown to have effects on Abeta amyloidosis through the modulation of host innate immunity in Parkinson's disease (Minter et al., 2017). It is admitted that a shift in microbiota can be influenced by various factors, including environment, species, and age. Therefore, we should conduct a double-blind, randomized, and placebo-controlled pilot study to determine the effects of A. muciniphila on rodents or humans and also to ensure experimental reproducibility. Moreover, although we can form a conclusion about the extraordinary achievement made by A. muciniphila and understand its molecular mechanism in treating obesity, we are unable to quantify the content of A. muciniphila that constitutes an effective dosage (Verhoog et al., 2019). Furthermore, A. muciniphila colonization is extensively different in different human groups; for instance,

\section{REFERENCES}

Allin, K. H., Tremaroli, V., Caesar, R., Jensen, B. A. H., Damgaard, M. T. F., and Bahl, I. (2018). Aberrant intestinal microbiota in individuals with prediabetes. Diabetologia 61, 810-820. doi: 10.1007/s00125-018-4550-1

Anhe, F. F., Pilon, G., Roy, D., Desjardins, Y., Levy, E., and Marette, A. (2016) Triggering Akkermansia with dietary polyphenols: a new weapon to combat the metabolic syndrome? Gut Microbes 7, 146-153. doi: 10.1080/19490976.2016. 1142036

Anhe, F. F., Schertzer, J. D., and Marette, A. (2019). Bacteria to alleviate metabolic syndrome. Nat. Med. 25, 1031-1033. doi: 10.1038/s41591-019-0516-1

Anonye, B. O. (2017). Commentary: dietary polyphenols promote growth of the gut bacterium Akkermansia muciniphila and attenuate high-fat dietinduced metabolic syndrome. Front. Immunol. 8:850. doi: 10.3389/fimmu.2017. 00850

Aron-Wisnewsky, J., Gaborit, B., Dutour, A., and Clement, K. (2013). Gut microbiota and non-alcoholic fatty liver disease: new insights. Clin. Microbiol. Infect. 19, 338-348. doi: 10.1111/1469-0691.12140

Baldwin, J., Collins, B., Wolf, P. G., Martinez, K., Shen, W., Chuang, C. C., et al. (2016). Table grape consumption reduces adiposity and markers of hepatic lipogenesis and alters gut microbiota in butter fat-fed mice. J. Nutr. Biochem. 27, 123-135. doi: 10.1016/j.jnutbio.2015.08.027

Bannerman, P., Guo, F., Chechneva, O., Burns, T., Zhu, X., Wang, Y., et al. (2018). Brain Nat8l knockdown suppresses spongiform leukodystrophy in an aspartoacylase-deficient canavan disease mouse model. Mol. Ther. 26, 793-800. doi: 10.1016/j.ymthe.2018.01.002

Belzer, C., Chia, L. W., Aalvink, S., Chamlagain, B., Piironen, V., and Knol, J. (2017). Microbial metabolic networks at the mucus layer lead to dietindependent butyrate and vitamin B12 production by intestinal symbionts. mBio 8:e00770-17. doi: 10.1128/mBio.00770-17

Belzer, C., and De Vos, W. M. (2012). Microbes inside-from diversity to function: the case of Akkermansia. ISME J. 6, 1449-1458. doi: 10.1038/ismej.2012.6

Brahe, L. K., Le Chatelier, E., Prifti, E., Pons, N., Kennedy, S., Hansen, T., et al. (2015). Specific gut microbiota features and metabolic markers in postmenopausal women with obesity. Nutr. Diabetes 5:e159. doi: 10.1038/nutd. 2015.9 residents of Southern China had a high rate of A. muciniphila, and over 12 different subtype strains resided in their guts (Guo et al., 2016).

\section{AUTHOR CONTRIBUTIONS}

YF conceived and designed the review. YX retrieved the literature and drafted the manuscript. NW, H-YT, SL, and CZ participated in the design of study and modification of English grammar. All authors read and approved the final manuscript.

\section{FUNDING}

The study was financially supported by grants from the Research Council of the University of Hong Kong (Project Codes: 104004092 and 104003919), Research Grants Committee of Hong Kong, HKSAR (Project Codes: 766211 and 17152116), Gala Family Trust (Project Code: 200007008), Government-Matching Grant Scheme (Project Code: 207060411), Donation of Vita Green Health Products Co., Ltd. (Project Code: 200007477), and Contract Research (Project Codes: 260007830 and 260007482). The funders had no role in study design, data collection and analysis, decision to publish, or preparation of the manuscript.

Brodmann, T., Endo, A., Gueimonde, M., Vinderola, G., Kneifel, W., De Vos, W. M., et al. (2017). Safety of novel microbes for human consumption: practical examples of assessment in the European Union. Front. Microbiol. 8:1725. doi: 10.3389/fmicb.2017.01725

Cani, P. D., Bibiloni, R., Knauf, C., Waget, A., Neyrinck, A. M., Delzenne, N. M., et al. (2008). Changes in gut microbiota control metabolic endotoxemiainduced inflammation in high-fat diet-induced obesity and diabetes in mice. Diabetes Metab. Res. Rev. 57, 1470-1481. doi: 10.2337/db07-1403

Cani, P. D., and de Vos, W. M. (2017). Next-generation beneficial microbes: the case of Akkermansia muciniphila. Front. Microbiol. 8:1765. doi: 10.3389/fmicb. 2017.01765

Cani, P. D., Osto, M., Geurts, L., and Everard, A. (2012). Involvement of gut microbiota in the development of low-grade inflammation and type 2 diabetes associated with obesity. Gut Microbes 3, 279-288. doi: 10.4161/gmic.19625

Cani, P. D., Possemiers, S., Van De Wiele, T., Guiot, Y., Everard, A., Rottier, O., et al. (2009). Changes in gut microbiota control inflammation in obese mice through a mechanism involving GLP-2-driven improvement of gut permeability. Gut 58, 1091-1103. doi: 10.1136/gut.2008.165886

Carding, S., Verbeke, K., Vipond, D. T., Corfe, B. M., and Owen, L. J. (2015). Dysbiosis of the gut microbiota in disease. Microb. Ecol. Health Dis. 26:26191. doi: 10.3402/mehd.v26.26191

Chambers, E. S., Preston, T., Frost, G., and Morrison, D. J. (2018). Role of gut microbiota-generated short-chain fatty acids in metabolic and cardiovascular health. Curr. Nutr. Rep. 7, 198-206. doi: 10.1007/s13668-018-0248-8

Chelakkot, C., Choi, Y., Kim, D. K., Park, H. T., Ghim, J., Kwon, Y., et al. (2018). Akkermansia muciniphila-derived extracellular vesicles influence gut permeability through the regulation of tight junctions. Exp. Mol. Med. 50:e450. doi: $10.1038 / \mathrm{emm} .2017 .282$

Chia, L. W., Hornung, B. V. H., Aalvink, S., Schaap, P. J., De Vos, W. M., Knol, J., et al. (2018). Deciphering the trophic interaction between Akkermansia muciniphila and the butyrogenic gut commensal Anaerostipes caccae using a metatranscriptomic approach. Antonie Van Leeuwenhoek 111, 859-873. doi: 10.1007/s10482-018-1040-x

Clemente, J. C., Ursell, L. K., Parfrey, L. W., and Knight, R. (2012). The impact of the gut microbiota on human health: an integrative view. Cell 148, 1258-1270. doi: $10.1016 /$ j.cell.2012.01.035 
Collado, M. C., Derrien, M., Isolauri, E., De Vos, W. M., and Salminen, S. (2007). Intestinal integrity and Akkermansia muciniphila, a mucin-degrading member of the intestinal microbiota present in infants, adults, and the elderly. Appl. Environ. Microbiol. 73, 7767-7770. doi: 10.1128/aem.01477-07

Collado, M. C., Isolauri, E., Laitinen, K., and Salminen, S. (2008). Distinct composition of gut microbiota during pregnancy in overweight and normalweight women. Am. J. Clin. Nutr. 88, 894-899. doi: 10.1093/ajcn/88.4.894

Collado, M. C., Isolauri, E., Laitinen, K., and Salminen, S. (2010). Effect of mother's weight on infant's microbiota acquisition, composition, and activity during early infancy: a prospective follow-up study initiated in early pregnancy. Am. J. Clin. Nutr. 92, 1023-1030. doi: 10.3945/ajcn.2010.29877

Collado, M. C., Laitinen, K., Salminen, S., and Isolauri, E. (2012). Maternal weight and excessive weight gain during pregnancy modify the immunomodulatory potential of breast milk. Pediatr. Res. 72, 77-85. doi: 10.1038/pr.2012.42

Cortez, R. V., Petry, T., Caravatto, P., Pessoa, R., Sanabani, S. S., Martinez, M. B., et al. (2018). Shifts in intestinal microbiota after duodenal exclusion favor glycemic control and weight loss: a randomized controlled trial. Surg. Obes. Relat. Dis. 14, 1748-1754. doi: 10.1016/j.soard.2018.07.021

Dao, M. C., Everard, A., Aron-Wisnewsky, J., Sokolovska, N., Prifti, E., Verger, E. O., et al. (2016). Akkermansia muciniphila and improved metabolic health during a dietary intervention in obesity: relationship with gut microbiome richness and ecology. Gut 65, 426-436. doi: 10.1136/gutjnl-2014308778

De La Cuesta-Zuluaga, J., Mueller, N. T., Corrales-Agudelo, V., Velasquez-Mejia, E. P., Carmona, J. A., Abad, J. M., et al. (2017). Metformin is associated with higher relative abundance of mucin-degrading Akkermansia muciniphila and several short-chain fatty acid-producing microbiota in the gut. Diabetes Care 40, 54-62. doi: 10.2337/dc16-1324

De Vos, W. M. (2017). microbe profile: Akkermansia muciniphila: a conserved intestinal symbiont that acts as the gatekeeper of our mucosa. Microbiology 163, 646-648. doi: 10.1099/mic.0.000444

Depommier, C., Everard, A., Druart, C., Plovier, H., Van Hul, M., Vieira-Silva, S., et al. (2019). Supplementation with Akkermansia muciniphila in overweight and obese human volunteers: a proof-of-concept exploratory study. Nat. Med. 25, 1096-1103. doi: 10.1038/s41591-019-0495-2

Derrien, M., Van Baarlen, P., Hooiveld, G., Norin, E., Muller, M., and De Vos, W. M. (2011). Modulation of mucosal immune response, tolerance, and proliferation in mice colonized by the mucin-degrader Akkermansia muciniphila. Front. Microbiol. 2:166. doi: 10.3389/fmicb.2011.00166

Derrien, M., Vaughan, E. E., Plugge, C. M., and de Vos, W. M. (2004). Akkermansia muciniphila gen. nov., sp. nov., a human intestinal mucin-degrading bacterium. Int. J. Syst. Evol. Microbiol. 54, 1469-1476. doi: 10.1099/ijs.0.02873-0

Dingemanse, C., Belzer, C., Van Hijum, S. A., Gunthel, M., Salvatori, D., Den Dunnen, J. T., et al. (2015). Akkermansia muciniphila and Helicobacter typhlonius modulate intestinal tumor development in mice. Carcinogenesis 36, 1388-1396. doi: 10.1093/carcin/bgv120

Duparc, T., Plovier, H., Marrachelli, V. G., Van Hul, M., Essaghir, A., Stahlman, M., et al. (2017). Hepatocyte MyD88 affects bile acids, gut microbiota and metabolome contributing to regulate glucose and lipid metabolism. Gut 66, 620-632. doi: 10.1136/gutjnl-2015-310904

Duranti, S., Ferrario, C., Van Sinderen, D., Ventura, M., and Turroni, F. (2017). Obesity and microbiota: an example of an intricate relationship. Genes Nutr. 12:18. doi: 10.1186/s12263-017-0566-2

Earley, H., Lennon, G., Balfe, A., Kilcoyne, M., Clyne, M., Joshi, L., et al. (2015). A preliminary study examining the binding capacity of Akkermansia muciniphila and Desulfovibrio spp., to colonic mucin in health and ulcerative colitis. PLoS One 10:e0135280. doi: 10.1371/journal.pone.0135280

Etxeberria, U., Arias, N., Boque, N., Macarulla, M. T., Portillo, M. P., Martinez, J. A., et al. (2015). Reshaping faecal gut microbiota composition by the intake of trans-resveratrol and quercetin in high-fat sucrose diet-fed rats. J. Nutr. Biochem. 26, 651-660. doi: 10.1016/j.jnutbio.2015.01.002

Everard, A., Belzer, C., Geurts, L., Ouwerkerk, J. P., Druart, C., Bindels, L. B., et al. (2013). Cross-talk between Akkermansia muciniphila and intestinal epithelium controls diet-induced obesity. Proc. Natl. Acad. Sci. U.S.A. 110, 9066-9071. doi: $10.1073 /$ pnas. 1219451110

Fukui, H. (2015). Gut microbiota and host reaction in liver diseases. Microorganisms 3, 759-791. doi: 10.3390/microorganisms3040759
Ganesh, B. P., Klopfleisch, R., Loh, G., and Blaut, M. (2013). Commensal Akkermansia muciniphila exacerbates gut inflammation in Salmonella Typhimurium-infected gnotobiotic mice. PLoS One 8:e74963. doi: 10.1371/ journal.pone.0074963

Geerlings, S. Y., Kostopoulos, I., De Vos, W. M., and Belzer, C. (2018). Akkermansia muciniphila in the human gastrointestinal tract: when, where, and how? Microorganisms 6:75. doi: 10.3390/microorganisms6030075

Gomez-Gallego, C., Pohl, S., Salminen, S., De Vos, W. M., and Kneifel, W. (2016). Akkermansia muciniphila: a novel functional microbe with probiotic properties. Benef. Microbes 7, 571-584. doi: 10.3920/BM2016.0009

Grander, C., Adolph, T. E., Wieser, V., Lowe, P., Wrzosek, L., Gyongyosi, B., et al. (2018). Recovery of ethanol-induced Akkermansia muciniphila depletion ameliorates alcoholic liver disease. Gut 67, 891-901. doi: 10.1136/gutjnl-2016313432

Greer, R. L., Dong, X., Moraes, A. C., Zielke, R. A., Fernandes, G. R., Peremyslova, E., et al. (2016). Akkermansia muciniphila mediates negative effects of IFNgamma on glucose metabolism. Nat. Commun. 7:13329. doi: 10.1038/ ncomms13329

Guo, X., Zhang, J., Wu, F., Zhang, M., Yi, M., and Peng, Y. (2016). Different subtype strains of Akkermansia muciniphila abundantly colonize in southern China. J. Appl. Microbiol. 120, 452-459. doi: 10.1111/jam.13022

Hanninen, A., Toivonen, R., Poysti, S., Belzer, C., Plovier, H., Ouwerkerk, J. P., et al. (2018). Akkermansia muciniphila induces gut microbiota remodelling and controls islet autoimmunity in NOD mice. Gut 67, 1445-1453. doi: 10.1136/ gutjnl-2017-314508

Hansen, C. H., Krych, L., Nielsen, D. S., Vogensen, F. K., Hansen, L. H., Sorensen, S. J., et al. (2012). Early life treatment with vancomycin propagates Akkermansia muciniphila and reduces diabetes incidence in the NOD mouse. Diabetologia 55, 2285-2294. doi: 10.1007/s00125-012-2564-7

Henning, S. M., Summanen, P. H., Lee, R. P., Yang, J., Finegold, S. M., Heber, D., et al. (2017). Pomegranate ellagitannins stimulate the growth of Akkermansia muciniphila in vivo. Anaerobe 43, 56-60. doi: 10.1016/j.anaerobe.2016.12.003

Huang, K., Wang, M. M., Kulinich, A., Yao, H. L., Ma, H. Y., Martinez, J. E., et al. (2015). Biochemical characterisation of the neuraminidase pool of the human gut symbiont Akkermansia muciniphila. Carbohydr. Res. 415, 60-65. doi: 10.1016/j.carres.2015.08.001

Kang, C. S., Ban, M., Choi, E. J., Moon, H. G., Jeon, J. S., Kim, D. K., et al. (2013). Extracellular vesicles derived from gut microbiota, especially Akkermansia muciniphila, protect the progression of dextran sulfate sodium-induced colitis. PLoS One 8:e76520. doi: 10.1371/journal.pone.0076520

Kelly, J. R., Kennedy, P. J., Cryan, J. F., Dinan, T. G., Clarke, G., and Hyland, N. P. (2015). Breaking down the barriers: the gut microbiome, intestinal permeability and stress-related psychiatric disorders. Front. Cell. Neurosci. 9:392. doi: 10. 3389/fncel.2015.00392

Khan, T. J., Ahmed, Y. M., Zamzami, M. A., Siddiqui, A. M., Khan, I., and Baothman, O. A. S. (2018). Atorvastatin treatment modulates the gut microbiota of the hypercholesterolemic patients. OMICS 22, 154-163. doi: 10. 1089/omi.2017.0130

Kim, J., Lee, H., An, J., Song, Y., Lee, C. K., Kim, K., et al. (2019). Alterations in gut microbiota by statin therapy and possible intermediate effects on hyperglycemia and hyperlipidemia. Front. Microbiol. 10:1947. doi: 10.3389/fmicb.2019. 01947

Leal-Diaz, A. M., Noriega, L. G., Torre-Villalvazo, I., Torres, N., AlemanEscondrillas, G., Lopez-Romero, P., et al. (2016). Aguamiel concentrate from Agave salmiana and its extracted saponins attenuated obesity and hepatic steatosis and increased Akkermansia muciniphila in C57BL6 mice. Sci. Rep. 6:34242. doi: 10.1038/srep34242

Ley, R. E., Backhed, F., Turnbaugh, P., Lozupone, C. A., Knight, R. D., and Gordon, J. I. (2005). Obesity alters gut microbial ecology. Proc. Natl. Acad. Sci. U.S.A. 102, 11070-11075.

Li, J., Lin, S., Vanhoutte, P. M., Woo, C. W., and Xu, A. (2016). Akkermansia muciniphila protects against atherosclerosis by preventing metabolic endotoxemia-induced inflammation in apoe-/- mice. Circulation 133, 2434-2446. doi: 10.1161/CIRCULATIONAHA.115.019645

Lin, P., Bach, M., Asquith, M., Lee, A. Y., Akileswaran, L., Stauffer, P., et al. (2014). HLA-B27 and human beta2-microglobulin affect the gut microbiota of transgenic rats. PLoS One 9:e105684. doi: 10.1371/journal.pone.0105684 
Liu, F., Ling, Z., Xiao, Y., Lv, L., Yang, Q., Wang, B., et al. (2017). Dysbiosis of urinary microbiota is positively correlated with type 2 diabetes mellitus. Oncotarget 8, 3798-3810. doi: 10.18632/oncotarget. 14028

Lopez-Siles, M., Enrich-Capo, N., Aldeguer, X., Sabat-Mir, M., Duncan, S. H., Garcia-Gil, L. J., et al. (2018). Alterations in the abundance and co-occurrence of Akkermansia muciniphila and Faecalibacterium prausnitzii in the colonic mucosa of inflammatory bowel disease subjects. Front. Cell. Infect. Microbiol. 8:281. doi: $10.3389 /$ fcimb. 2018.00281

Lukovac, S., Belzer, C., Pellis, L., Keijser, B. J., De Vos, W. M., and Montijn, R. C. (2014). Differential modulation by Akkermansia muciniphila and Faecalibacterium prausnitzii of host peripheral lipid metabolism and histone acetylation in mouse gut organoids. mBio 5:e01438-14. doi: 10.1128/mBio. 01438-14

Medina-Vera, I., Sanchez-Tapia, M., Noriega-Lopez, L., Granados-Portillo, O., Guevara-Cruz, M., Flores-Lopez, A., et al. (2019). A dietary intervention with functional foods reduces metabolic endotoxaemia and attenuates biochemical abnormalities by modifying faecal microbiota in people with type 2 diabetes. Diabetes Metab. 45, 122-131. doi: 10.1016/j.diabet.2018.09.004

Meng, X., Li, S., Li, Y., Gan, R. Y., and Li, H. B. (2018). Gut microbiota’s relationship with liver disease and role in hepatoprotection by dietary natural Products and probiotics. Nutrients 10:1457. doi: 10.3390/nu10101457

Minter, M. R., Hinterleitner, R., Meisel, M., Zhang, C., Leone, V., Zhang, X., et al. (2017). Antibiotic-induced perturbations in microbial diversity during postnatal development alters amyloid pathology in an aged APPSWE/PS1DeltaE9 murine model of Alzheimer's disease. Sci. Rep. 7:10411. doi: 10.1038/s41598017-11047-w

Nehra, V., Allen, J. M., Mailing, L. J., Kashyap, P. C., and Woods, J. A. (2016). Gut Microbiota: modulation of host physiology in obesity. Physiology (Bethesda) 31, 327-335. doi: 10.1152/physiol.00005.2016

Ottman, N., Davids, M., Suarez-Diez, M., Boeren, S., Schaap, P. J., and Martins Dos Santos, V. A. P. (2017a). Genome-scale model and omics analysis of metabolic capacities of Akkermansia muciniphila reveal a preferential mucin-degrading lifestyle. Appl. Environ. Microbiol. 83:e01014-17. doi: 10.1128/AEM.01 014-17

Ottman, N., Geerlings, S. Y., Aalvink, S., De Vos, W. M., and Belzer, C. (2017b). Action and function of Akkermansia muciniphila in microbiome ecology, health and disease. Best Pract. Res. Clin. Gastroenterol. 31, 637-642. doi: 10.1016/j.bpg. 2017.10.001

Ottman, N., Huuskonen, L., Reunanen, J., Boeren, S., Klievink, J., Smidt, H., et al. (2016). Characterization of outer membrane proteome of Akkermansia muciniphila reveals sets of novel proteins exposed to the human intestine. Front. Microbiol. 7:1157. doi: 10.3389/fmicb.2016.01157

Ottman, N., Reunanen, J., Meijerink, M., Pietila, T. E., Kainulainen, V., Klievink, J., et al. (2017c). Pili-like proteins of Akkermansia muciniphila modulate host immune responses and gut barrier function. PLoS One 12:e0173004. doi: 10. 1371/journal.pone.0173004

Ouwerkerk, J. P., Aalvink, S., Belzer, C., and De Vos, W. M. (2017). Preparation and preservation of viable Akkermansia muciniphila cells for therapeutic interventions. Benef. Microbes 8, 163-169. doi: 10.3920/BM2016.0096

Ouwerkerk, J. P., Van Der Ark, K. C. H., Davids, M., Claassens, N. J., Finestra, T. R., De Vos, W. M., et al. (2016). Adaptation of Akkermansia muciniphila to the oxic-anoxic interface of the mucus layer. Appl. Environ. Microbiol. 82, 6983-6993. doi: 10.1128/AEM.01641-16

Ozdal, T., Sela, D. A., Xiao, J., Boyacioglu, D., Chen, F., and Capanoglu, E. (2016). The reciprocal interactions between polyphenols and gut microbiota and effects on bioaccessibility. Nutrients 8:78. doi: 10.3390/nu8020078

Ozkul, C., Yalinay, M., Karakan, T., and Yilmaz, G. (2017). Determination of certain bacterial groups in gut microbiota and endotoxin levels in patients with nonalcoholic steatohepatitis. Turk. J. Gastroenterol. 28, 361-369. doi: 10.5152/ tjg.2017.17033

Palleja, A., Kashani, A., Allin, K. H., Nielsen, T., Zhang, C., Li, Y., et al. (2016). Roux-en-Y gastric bypass surgery of morbidly obese patients induces swift and persistent changes of the individual gut microbiota. Genome Med. 8:67. doi: 10.1186/s13073-016-0312-1

Pascale, A., Marchesi, N., Govoni, S., Coppola, A., and Gazzaruso, C. (2019). The role of gut microbiota in obesity, diabetes mellitus, and effect of metformin: new insights into old diseases. Curr. Opin. Pharmacol. 49, 1-5. doi: 10.1016/j.coph. 2019.03.011
Pierre, J. F., Heneghan, A. F., Feliciano, R. P., Shanmuganayagam, D., Roenneburg, D. A., Krueger, C. G., et al. (2013). Cranberry proanthocyanidins improve the gut mucous layer morphology and function in mice receiving elemental enteral nutrition. JPEN J. Parenter. Enteral. Nutr. 37, 401-409. doi: 10.1177/ 0148607112463076

Pleasure, D., Guo, F., Chechneva, O., Bannerman, P., Mcdonough, J., and Burns, T. (2018). Pathophysiology and treatment of canavan disease. Neurochem. Res. doi: 10.1007/s11064-018-2693-6

Plovier, H., Everard, A., Druart, C., Depommier, C., Van Hul, M., Geurts, L., et al. (2017). A purified membrane protein from Akkermansia muciniphila or the pasteurized bacterium improves metabolism in obese and diabetic mice. Nat. Med. 23, 107-113. doi: $10.1038 / \mathrm{nm} .4236$

Remely, M., Hippe, B., Zanner, J., Aumueller, E., Brath, H., and Haslberger, A. G. (2016). Gut microbiota of obese, type 2 diabetic individuals is enriched in Faecalibacterium prausnitzii, Akkermansia muciniphila and Peptostreptococcus anaerobius after weight loss. Endocr. Metab. Immune Disord. Drug Targets 16, 99-106. doi: 10.2174/1871530316666160831093813

Reunanen, J., Kainulainen, V., Huuskonen, L., Ottman, N., Belzer, C., Huhtinen, H., et al. (2015). Akkermansia muciniphila adheres to enterocytes and strengthens the integrity of the epithelial cell layer. Appl. Environ. Microbiol. 81, 3655-3662. doi: 10.1128/AEM.04050- 14

Roopchand, D. E., Carmody, R. N., Kuhn, P., Moskal, K., Rojas-Silva, P., Turnbaugh, P. J., et al. (2015). dietary polyphenols promote growth of the gut bacterium Akkermansia muciniphila and attenuate high-fat diet-induced metabolic syndrome. Diabetes Metab. Res. Rev. 64, 2847-2858. doi: 10.2337/ db14-1916

Roshanravan, N., Mahdavi, R., Alizadeh, E., Ghavami, A., Rahbar Saadat, Y., Mesri Alamdari, N., et al. (2017). The effects of sodium butyrate and inulin supplementation on angiotensin signaling pathway via promotion of Akkermansia muciniphila abundance in type 2 diabetes; a randomized, doubleblind, placebo-controlled trial. J. Cardiovasc. Thorac. Res. 9, 183-190. doi: 10. $15171 /$ jcvtr.2017.32

Santacruz, A., Collado, M. C., Garcia-Valdes, L., Segura, M. T., Martin-Lagos, J. A., Anjos, T., et al. (2010). Gut microbiota composition is associated with body weight, weight gain and biochemical parameters in pregnant women. $\mathrm{Br}$. J. Nutr. 104, 83-92. doi: 10.1017/S0007114510000176

Schneeberger, M., Everard, A., Gomez-Valades, A. G., Matamoros, S., Ramirez, S., Delzenne, N. M., et al. (2015). Akkermansia muciniphila inversely correlates with the onset of inflammation, altered adipose tissue metabolism and metabolic disorders during obesity in mice. Sci. Rep. 5:16643. doi: 10.1038/ srep16643

Sergey, S. S., Golovchenko, N., and Schaf, B. (2017). Bryan schaf NLRP6 protects Il10 : mice from colitis by limiting colonization of Akkermansia muciniphila. Cell Rep. 19, 733-745. doi: 10.1016/j.celrep.2017.03.080

Shen, J., Tong, X., Sud, N., Khound, R., Song, Y., Maldonado-Gomez, M. X., et al. (2016). Low-density lipoprotein receptor signaling mediates the triglyceride-Lowering action of Akkermansia muciniphila in genetic-induced hyperlipidemia. Arterioscler. Thromb. Vasc. Biol. 36, 1448-1456. doi: 10.1161/ ATVBAHA.116.307597

Shen, W., Shen, M., Zhao, X., Zhu, H., Yang, Y., Lu, S., et al. (2017). Anti-obesity effect of capsaicin in mice fed with high-fat diet is associated with an increase in population of the gut bacterium Akkermansia muciniphila. Front. Microbiol. 8:272. doi: $10.3389 /$ fmicb.2017.00272

Shin, N. R., Lee, J. C., Lee, H. Y., Kim, M. S., Whon, T. W., Lee, M. S., et al. (2014). An increase in the Akkermansia spp. population induced by metformin treatment improves glucose homeostasis in diet-induced obese mice. Gut 63, 727-735. doi: 10.1136/gutjnl-2012-303839

Sonnenburg, J. L., and Backhed, F. (2016). Diet-microbiota interactions as moderators of human metabolism. Nature 535, 56-64. doi: 10.1038/ nature 18846

Spanogiannopoulos, P., Bess, E. N., Carmody, R. N., and Turnbaugh, P. J. (2016). The microbial pharmacists within us: a metagenomic view of xenobiotic metabolism. Nat. Rev. Microbiol. 14, 273-287. doi: 10.1038/nrmicro. 2016.17

Stenman, L. K., Burcelin, R., and Lahtinen, S. (2016). Establishing a causal link between gut microbes, body weight gain and glucose metabolism in humans - towards treatment with probiotics. Benef. Microbes 7, 11-22. doi: 10.3920/ BM2015.0069 
Swidsinski, A., Dorffel, Y., Loening-Baucke, V., Theissig, F., Ruckert, J. C., Ismail, M., et al. (2011). Acute appendicitis is characterised by local invasion with Fusobacterium nucleatum/necrophorum. Gut 60, 34-40. doi: 10.1136/gut.2009. 191320

Turnbaugh, P. J., Backhed, F., Fulton, L., and Gordon, J. I. (2008). Diet-induced obesity is linked to marked but reversible alterations in the mouse distal gut microbiome. Cell Host Microbe 3, 213-223. doi: 10.1016/j.chom.2008. 02.015

Van Best, N., Jansen, P. L., and Rensen, S. S. (2015). The gut microbiota of nonalcoholic fatty liver disease: current methods and their interpretation. Hepatol. Int. 9, 406-415. doi: 10.1007/s12072-015-9640-2

Van Der Ark, K. C. H., Aalvink, S., Suarez-Diez, M., Schaap, P. J., De Vos, W. M., and Belzer, C. (2018). Model-driven design of a minimal medium for Akkermansia muciniphila confirms mucus adaptation. Microb. Biotechnol. 11, 476-485. doi: 10.1111/1751-7915.13033

Van Herreweghen, F., De Paepe, K., Roume, H., Kerckhof, F. M., and Van De Wiele, T. (2018). Mucin degradation niche as a driver of microbiome composition and Akkermansia muciniphila abundance in a dynamic gut model is donor independent. FEMS Microbiol. Ecol. 94:fiy186. doi: 10.1093/femsec/ fiy 186

Verhoog, S., Taneri, P. E., Roa Diaz, Z. M., Marques-Vidal, P., Troup, J. P., and Bally, L. (2019). Dietary factors and modulation of bacteria strains of Akkermansia muciniphila and Faecalibacterium prausnitzii: a systematic review. Nutrients 11:1565. doi: 10.3390/nu11071565

Walker, J. M., Eckardt, P., Aleman, J. O., Da Rosa, J. C., Liang, Y., Iizumi, T., et al. (2019). The effects of trans-resveratrol on insulin resistance, inflammation, and microbiota in men with the metabolic syndrome: a pilot randomized, placebo-controlled clinical trial. J. Clin. Transl. Res. 4, 122-135.

Wang, J., and Jia, H. (2016). Metagenome-wide association studies: fine-mining the microbiome. Nat. Rev. Microbiol. 14, 508-522. doi: 10.1038/nrmicro.20 16.83

Wang, L. (2011). The relative abundance of the mucolytic bacterium Akkermansia muciniphila and Bifidobacterium spp. is lower in feces of children with autism. Appl. Environ. Microbiol. 77, 6718-6721. doi: 10.1128/aem.052 $12-11$

Wang, Y., Liu, Q., Xu, Y., Zhang, Y., Lv, Y., Tan, Y., et al. (2016). Ginsenoside Rg1 protects against oxidative stress-induced neuronal apoptosis through myosin IIA-actin related cytoskeletal reorganization. Int. J. Biol. Sci. 12, 1341-1356. doi: 10.7150/ijbs.15992

Wang, Y., Xu, Y., Liu, Q., Zhang, Y., Gao, Z., Yin, M., et al. (2017). Myosin IIArelated actomyosin contractility mediates oxidative stress-induced neuronal apoptosis. Front. Mol. Neurosci. 10:75. doi: 10.3389/fnmol.2017.00075

Wu, W., Lv, L., Shi, D., Ye, J., Fang, D., Guo, F., et al. (2017). Protective effect of Akkermansia muciniphila against immune-mediated liver injury in a mouse model. Front. Microbiol. 8:1804. doi: 10.3389/fmicb.2017.01804
Yassour, M., Lim, M. Y., Yun, H. S., Tickle, T. L., Sung, J., Song, Y. M., et al. (2016). Sub-clinical detection of gut microbial biomarkers of obesity and type 2 diabetes. Genome Med. 8:17. doi: 10.1186/s13073-016-0271-6

Zhai, K., Tang, Y., Zhang, Y., Li, F., Wang, Y., Cao, Z., et al. (2015). NMMHC IIA inhibition impedes tissue factor expression and venous thrombosis via Akt/GSK3 $\beta$-NF- $\mathrm{kB}$ signalling pathways in the endothelium. Thromb. Haemost. 114, 173-185. doi: 10.1160/th14-10-0880

Zhai, Q., Feng, S., Arjan, N., and Chen, W. (2018). A next generation probiotic, Akkermansia muciniphila. Crit. Rev. Food Sci. Nutr. 59, 3227-3236. doi: 10. 1080/10408398.2018.1517725

Zhang, H., Sparks, J. B., Karyala, S. V., Settlage, R., and Luo, X. M. (2015). Host adaptive immunity alters gut microbiota. ISME J. 9, 770-781. doi: 10.1038/ ismej.2014.165

Zhang, L., Carmody, R. N., Kalariya, H. M., Duran, R. M., Moskal, K., Poulev, A., et al. (2018). Grape proanthocyanidin-induced intestinal bloom of Akkermansia muciniphila is dependent on its baseline abundance and precedes activation of host genes related to metabolic health. J. Nutr. Biochem. 56, 142-151. doi: 10.1016/j.jnutbio.2018.02.009

Zhang, L., Wang, Y., Xiayu, X., Shi, C., Chen, W., Song, N., et al. (2017). Altered gut microbiota in a mouse model of Alzheimer's disease. J. Alzheimers Dis. 60, 1241-1257. doi: 10.3233/JAD- 170020

Zhang, S., Rasai, A., Wang, Y., Xu, J., Bannerman, P., Erol, D., et al. (2018). The Stem cell factor Sox2 is a positive timer of oligodendrocyte development in the postnatal murine spinal cord. Mol. Neurobiol. 55, 9001-9015. doi: 10.1007/ s12035-018-1035-7

Zhang, T., Li, Q., Cheng, L., Buch, H., and Zhang, F. (2019). Akkermansia muciniphila is a promising probiotic. Microb. Biotechnol. 12, 1109-1125. doi: 10.1111/1751-7915.13410

Zhao, S., Liu, W., Wang, J., Shi, J., Sun, Y., Wang, W., et al. (2017). Akkermansia muciniphila improves metabolic profiles by reducing inflammation in chow diet-fed mice. J. Mol. Endocrinol. 58, 1-14. doi: 10.1530/jme-16-0054

Zheng, H., Liang, H., Wang, Y., Miao, M., Shi, T., Yang, F., et al. (2016). Altered gut microbiota composition associated with eczema in infants. PLoS One 11:e0166026. doi: 10.1371/journal.pone.0166026

Conflict of Interest: The authors declare that the research was conducted in the absence of any commercial or financial relationships that could be construed as a potential conflict of interest.

Copyright (c) $2020 \mathrm{Xu}$, Wang, Tan, Li, Zhang and Feng. This is an open-access article distributed under the terms of the Creative Commons Attribution License (CC BY). The use, distribution or reproduction in other forums is permitted, provided the original author(s) and the copyright owner(s) are credited and that the original publication in this journal is cited, in accordance with accepted academic practice. No use, distribution or reproduction is permitted which does not comply with these terms. 